\title{
Computation of the Néron-Tate Height on Elliptic Curves
}

\author{
By Heinz M. Tschöpe and Horst G. Zimmer
}

For Daniel Shanks on the occasion of his 70 th birthday

\begin{abstract}
Using Néron's reduction theory and a method of Tate, we develop a procedure for calculating the local and global Néron-Tate height on an elliptic curve over the rationals. The procedure is illustrated by means of two examples of Silverman and is then applied to calculate the global Néron-Tate height of a series of rank-one curves of Bremner-Cassels and of a series of rank-two curves of Selmer. In the latter case, the regulator is also computed, and a conjecture of S. Lang is investigated numerically.

In dealing with the arithmetic of elliptic curves $E$ over a global field $K$, the task arises of computing the Néron-Tate height on the group $E(K)$ of rational points of $E$ over $K$. Solving this task in an efficient manner is important, for instance, in view of calculations concerning the Birch and Swinnerton-Dyer conjecture (see [2]) or of the conjectures of Serge Lang [6]. The purpose of this note is to suggest a procedure for performing the necessary calculations.
\end{abstract}

1. Multiplication Formulas. Let the elliptic curve $E$ over any field $K$ be defined by a generalized Weierstrass equation

$$
y^{2}+a_{1} x y+a_{3} y=x^{3}+a_{2} x^{2}+a_{4} x+a_{6} \quad\left(a_{i} \in K\right) .
$$

As usual, we introduce the quantities (see [10], [11])

$$
\begin{aligned}
& b_{2}=a_{1}^{2}+4 a_{2}, \quad b_{4}=a_{1} a_{3}+2 a_{4}, \quad b_{6}=a_{3}^{2}+4 a_{6}, \\
& b_{8}=a_{1}^{2} a_{6}-a_{1} a_{3} a_{4}+4 a_{2} a_{6}+a_{2} a_{3}^{2}-a_{4}^{2}, \\
& c_{4}=b_{2}^{2}-24 b_{4}, \quad c_{6}=-b_{2}^{3}+36 b_{2} b_{4}-216 b_{6},
\end{aligned}
$$

and the discriminant

$$
\Delta=-b_{2}^{2} b_{8}-8 b_{4}^{3}-27 b_{6}^{2}+9 b_{2} b_{4} b_{6} \neq 0,
$$

as well as the absolute invariant

$$
j=c_{4}^{3} / \Delta
$$

belonging to $E$ over $K$.

The fact that $E$ is nonsingular implies the nonvanishing of the partial derivatives of the polynomial

$$
F(x, y)=y^{2}+a_{1} x y+a_{3} y-x^{3}-a_{2} x^{2}-a_{4} x-a_{6}
$$

Received May 19, 1986; revised June 30, 1986.

1980 Mathematics Subject Classification. Primary 14-04, 14H45, 14K07, 14K15; Secondary 10B10, $14 \mathrm{G} 20,14 \mathrm{G} 25,14 \mathrm{H} 25$. 
at every rational point $P \in E(K)$ :

$$
\left(\frac{\partial F}{\partial x}(P), \frac{\partial F}{\partial y}(P)\right) \neq(0,0) .
$$

The addition law in the additive Abelian group $E(K)$ of rational points on $E$ over $K$ is given by the following formulas:

For $P=\left(x_{P}, y_{P}\right), Q=\left(x_{Q}, y_{Q}\right) \in E(K)$, denote the sum by $P+Q=$ $\left(x_{P+Q}, y_{P+Q}\right)$. Then,

$$
\begin{aligned}
& x_{P+Q}=-\left(x_{P}+x_{Q}\right)+\left(\frac{y_{P}-y_{Q}}{x_{P}-x_{Q}}\right)^{2}+a_{1}\left(\frac{y_{P}-y_{Q}}{x_{P}-x_{Q}}\right)-a_{2}, \\
& y_{P+Q}=\frac{y_{P}-y_{Q}}{x_{P}-x_{Q}}\left(x_{P}-x_{P+Q}\right)-a_{1} x_{P+Q}-a_{3}-y_{P} \quad \text { if } P \neq Q
\end{aligned}
$$

and

$$
\begin{array}{cc}
x_{2 P}=-2 x_{P}+t_{P}^{2}+a_{1} t_{P}-a_{2}, \quad y_{2 P}=t_{P}\left(x_{P}-x_{2 P}\right)-a_{1} x_{2 P}-a_{3}-y_{P} \\
\text { for } t_{P}=\frac{3 x_{P}^{2}+2 a_{2} x_{P}+a_{4}-a_{1} y_{P}}{2 y_{P}+a_{1} x_{P}+a_{3}} & \text { if } P=Q .
\end{array}
$$

Generalizing classical formulas (see [3], [4], [15]), we obtain

Proposition 1. For a rational point $P \in E(K)$ and an $r \in \mathbf{N}$, the r-fold rational point has coordinates

$$
r P=\left(x_{r P}, y_{r P}\right)=\left(\frac{\Phi_{r}(P)}{\Psi_{r}^{2}(P)}, \frac{\Omega_{r}(P)}{\Psi_{r}^{3}(P)}\right),
$$

where $\Phi_{r}, \Psi_{r}$, and $2 \Omega_{r}$ are polynomials in $x$ and $y$ with coefficients in $\mathbf{Z}\left[a_{1}, a_{2}, a_{3}, a_{4}, a_{6}\right]$ given by the following recursion formulas:

$$
\begin{aligned}
& \Phi_{1}=x, \quad \Phi_{2}=x^{4}-b_{4} x^{2}-2 b_{6} x-b_{8}, \\
& \Omega_{1}=y, \quad \Psi_{0}=0, \quad \Psi_{1}=1, \Psi_{2}=2 y+a_{1} x+a_{3}, \\
& \Psi_{3}=3 x^{4}+b_{2} x^{3}+3 b_{4} x^{2}+3 b_{6} x+b_{8}, \\
& \Psi_{4}=\Psi_{2}\left[2 x^{6}+b_{2} x^{5}+5 b_{4} x^{4}+10 b_{6} x^{3}+10 b_{8} x^{2}+\left(b_{2} b_{8}-b_{4} b_{6}\right) x+b_{4} b_{8}-b_{6}^{2}\right]
\end{aligned}
$$

and for $r \geqslant 2$,

$$
\begin{aligned}
& \Phi_{r}=x \Psi_{r}^{2}-\Psi_{r-1} \Psi_{r+1}, \\
& 2 \Psi_{2} \Omega_{r}=\Psi_{r-1}^{2} \Psi_{r+2}-\Psi_{r-2} \Psi_{r+1}^{2}-\Psi_{2} \Psi_{r}\left[a_{1} \Phi_{r}+a_{3} \Psi_{r}^{2}\right], \\
& \Psi_{2 r+1}=\Psi_{r}^{3} \Psi_{r+2}-\Psi_{r-1} \Psi_{r+1}^{3}, \\
& \Psi_{2} \Psi_{2 r}=\Psi_{r}\left[\Psi_{r-1}^{2} \Psi_{r+2}-\Psi_{r-2} \Psi_{r+1}^{2}\right] .
\end{aligned}
$$

Moreover, $\Phi_{r}$, as a polynomial in $x$, has degree $r^{2}$ and leading coefficient 1 , whereas $\Psi_{r}\left(\right.$ resp. $\left.\Psi_{2}^{-1} \Psi_{r}\right)$, as a polynomial in $x$, has degree $\left(r^{2}-1\right) / 2\left(\right.$ resp. $\left.\left(r^{2}-4\right) / 2\right)$ and leading coefficient $r$ (resp. $r / 2$ ) provided that $r$ is odd (resp. even). If we assign the weight 2, 3 or $i$ to $x, y$ or $a_{i}$, then each term of $\Phi_{r}$ has weight $2 r^{2}$ and each term of 
$\Psi_{r}$ (resp. $\left.\Psi_{2}^{-1} \Psi_{r}\right)$ has weight $r^{2}-1$ (resp. $\left.r^{2}-4\right)$. The coefficients of $\Phi_{r}, \Psi_{r}$, as polynomials in $x, y$, belong already to $\mathbf{Z}\left[b_{2}, b_{4}, b_{6}, b_{8}\right]$.

From Proposition 1, one derives the following

Corollary. For $r \in \mathbf{N}$, we put $\Psi_{-r}=-\Psi_{r}$. Then, for $r, n \in \mathbf{N}$, we have

$$
\Psi_{r n}^{2}(P)=\Psi_{n}^{2 r^{2}}(P) \Psi_{r}^{2}(n P)
$$

and, more generally,

$$
\Psi_{m^{n}}^{2}(P)=\prod_{\nu=1}^{n} \Psi_{m}^{\left.2 m^{2(n-\nu}\right)}\left(m^{\nu-1} P\right)
$$

Furthermore,

$$
\begin{gathered}
x_{r P}-x_{n P}=-\frac{\Psi_{r+n}(P) \Psi_{r-n}(P)}{\Psi_{r}^{2}(P) \Psi_{n}^{2}(P)} \\
x_{r P}-x_{r Q}=(-1)^{r+1} \frac{\Psi_{r}(P+Q) \Psi_{r}(P-Q)}{\Psi_{r}^{2}(P) \Psi_{r}^{2}(Q)}\left(x_{P}-x_{Q}\right)^{r^{2}}
\end{gathered}
$$

and finally, for $r \in \mathbf{N}_{0}$,

$$
\Phi_{2}\left(2^{r} P\right)=\Phi_{2^{r+1}}(P) \Psi_{2^{r}}^{-8}(P), \quad \Psi_{2}^{2}\left(2^{r} P\right)=\Psi_{2^{r+1}}^{2}(P) \Psi_{2^{r}}^{-8}(P) .
$$

These formulas will be needed in the sequel.

2. Reduction Theory. Now let the elliptic curve $E$ be defined by (E) over a complete field $K$ with respect to a discrete normalized additive valuation $v$, and suppose that the corresponding residue field $\tilde{K}$ of $K$ is perfect. We assume the equation defining $E$ over $K$ to be minimal with respect to the valuation $v$ (see [11]). Reducing $E$ modulo $v$ yields a cubic curve

$$
\tilde{y}^{2}+\tilde{a}_{1} \tilde{x} \tilde{y}+\tilde{a}_{3} \tilde{y}=\tilde{x}^{3}+\tilde{a}_{2} \tilde{x}^{2}+\tilde{a}_{4} \tilde{x}+\tilde{a}_{6} \quad\left(\tilde{a}_{i} \in \tilde{K}\right)
$$

over $\tilde{K}$ with discriminant $\tilde{\Delta}$. If $\tilde{\Delta} \neq 0$, i.e., $v(\Delta)=0$, then $\tilde{E}$ is an elliptic curve over $\tilde{K}$, and $E$ has good reduction at $v$. If, however, $\tilde{\Delta}=0$, i.e., $v(\Delta)>0$, then $\tilde{E}$ is a rational curve over $\tilde{K}$, and $E$ has bad reduction at $v$. In the latter case, $E$ is said to have multiplicative reduction or additive reduction modulo $v$, according as $v\left(c_{4}\right)=0$ or $v\left(c_{4}\right)>0$, respectively.

Denote by $E_{0}(K)$ the set of points in $E(K)$ whose image under the reduction map modulo $v$,

$$
\rho: E(K) \rightarrow \tilde{E}(\tilde{K})
$$

is a nonsingular point on $\tilde{E}$ over $\tilde{K}$. Then, $E_{0}(K)$ is a subgroup of finite index in $E(K)$. Further, the set

$$
E_{1}(K)=\left\{P=\left(x_{P}, y_{P}\right) \in E(K) \mid v\left(x_{P}\right) \leqslant-2, v\left(y_{P}\right) \leqslant-3\right\}
$$


is a subgroup of $E_{0}(K)$, and the restriction $\rho_{0}$ to $E_{0}(K)$ of the reduction map $\rho$ induces an injective homomorphism of the factor group

$$
\tilde{\rho}_{0}: E_{0}(K) / E_{1}(K) \rightarrow \tilde{E}_{0}(\tilde{K})
$$

to the nonsingular part $\tilde{E}_{0}(\tilde{K})$ of $\tilde{E}(\tilde{K})$.

We shall use the following result (see [11]).

Proposition 2. The above groups satisfy

$$
\begin{aligned}
& E(K)=E_{0}(K) \text { if } E \text { has good reduction at } v, \\
& \#\left(E(K) / E_{0}(K)\right) \text { divides } v(j) \text { if } E \text { has multiplicative reduction at } v,
\end{aligned}
$$

and

$$
\#\left(E(K) / E_{0}(K)\right) \leqslant 4 \text { if } E \text { has additive reduction at } v
$$

3. Definition of Height Functions. Now let $K$ be a global field, that is, an algebraic number field or a function field of finite transcendence degree over its field of constants $k$. Then $K$ possesses a complete set $M_{K}$ of nonequivalent additive valuations $v$ satisfying the sum formula

$$
\sum_{v \in M_{K}} \lambda_{v} v(c)=0 \quad \text { for } 0 \neq c \in K
$$

with some positive multiplicities $\lambda_{v} \in \mathbf{R}$ (cf. [7], [13]).

For an elliptic curve $E$ over $K$, given by the Weierstrass equation (E), we introduce the quantities

$$
\mu_{v}=\min \left\{v\left(b_{2}\right), \frac{1}{2} v\left(b_{4}\right), \frac{1}{3} v\left(b_{6}\right), \frac{1}{4} v\left(b_{8}\right)\right\} .
$$

Let $P=\left(x_{P}, y_{P}\right) \in E(K)$ be any rational point and $\mathcal{O}=(\infty, \infty)$ designate the point at infinity. Then we define the local Weil height on $E(K)$ with respect to $v$ by setting

$$
d_{v}(P)= \begin{cases}-\frac{1}{2} \min \left\{\mu_{v}, v\left(x_{P}\right)\right\} & \text { if } P \neq \mathcal{O}, \\ -\frac{1}{2} \mu_{v} & \text { if } P=\mathcal{O} .\end{cases}
$$

Then the global Weil height on $E(K)$ is simply the sum, with multiplicities, over the local Weil heights

$$
d(P)=\sum_{v \in M_{K}} \lambda_{v} d_{v}(P)
$$

(see [13]).

In order to define the global Néron-Tate height on $E(K)$, we proceed in the same way as with the global Weil height. However, before introducing the local Néron-Tate height on $E(K)$, we need some estimates.

Proposition 3. The local Weil height on $E(K)$ satisfies the following estimates:

$$
\begin{aligned}
\frac{1}{2}\left(6 \mu_{v}-v(\Delta)\right)+5 \alpha_{v} \leqslant & d_{v}(P+Q)+d_{v}(P-Q) \\
& -2 d_{v}(P)-2 d_{v}(Q)-v\left(x_{P}-x_{Q}\right) \\
& \leqslant-2 \alpha_{v} \quad \text { if } P, Q, P \pm Q \neq \mathcal{O},
\end{aligned}
$$


and

$$
\begin{aligned}
\frac{1}{2}\left(6 \mu_{v}-v(\Delta)\right)+4 \alpha_{v} & \leqslant d_{v}(2 P)-4 d_{v}(P)-\frac{1}{2} v\left(\Psi_{2}^{2}(P)\right) \\
& \leqslant-\frac{3}{2} \alpha_{v} \quad \text { if } 2 P \neq \mathcal{O},
\end{aligned}
$$

where the constant $\alpha_{v}$ can be chosen to be 0 or $-\log 2$ according as the valuation $v$ of $K$ is discrete or archimedean, respectively (see [13]).

These estimates are obtained as generalizations of those given in [13], [14]. At the same time, they sharpen those cited.

Remark 1. It is interesting to note that the authors of [2] suggested that a sharpening of the estimates in [13], [14] should be possible. Proposition 3 appears to be a step in this direction.

Employing (10), the inequalities (11) can be further generalized.

COROLlaRY. For any $m \in \mathbf{N}$, there are (recursively computable) nonnegative constants $c_{1, m}, c_{2, m} \in \mathbf{R}$ depending on $E, K$, and $v$ such that, given an arbitrary point $P \in E(K)$ with $m P \neq \mathcal{O}$, we have

$$
c_{1, m} \leqslant d_{v}(m P)-m^{2} d_{v}(P)-\frac{1}{2} v\left(\Psi_{m}^{2}(P)\right) \leqslant c_{2, m} .
$$

We are now in a position to define the local Néron-Tate height on $E(K)$ with respect to $v$. Let $m, n \in \mathbf{N}$ and $m \geqslant 2$. Then, for a rational point $P \in E(K)$ such that $m^{n} P \neq \mathcal{O}$ for each $n \in \mathbf{N}$, we define the local Neron-Tate height of $P$ with respect to $v$ by the limit formula

$$
\delta_{v, m}(P)=\lim _{n \rightarrow \infty}\left\{\frac{d_{v}\left(m^{n} P\right)}{m^{2 n}}-\frac{1}{2} \frac{v\left(\Psi_{m^{n}}^{2}(P)\right)}{m^{2 n}}\right\}+\frac{1}{12} v(\Delta) .
$$

Proposition 4. For an elliptic curve $E$ defined by a Weierstrass equation (E) over a global field $K$ and any valuation $v$ of $K$, the function $\delta_{v, m}$, defined by (12) on the rational point group $E(K)$, exists, is independent of the choice of $m \in \mathbf{N}$, so that $\delta_{v, m}=\delta_{v}$, and fulfills the relations

$$
\delta_{v}(P+Q)+\delta_{v}(P-Q)-2 \delta_{v}(P)-2 \delta_{v}(Q)-v\left(x_{P}-x_{Q}\right)+\frac{1}{6} v(\Delta)=0
$$

for any two points $P=\left(x_{P}, y_{P}\right), Q=\left(x_{Q}, y_{Q}\right) \in E(K)$ such that $P, Q, P \pm Q \neq \mathcal{O}$, and

$$
\delta_{v}(r P)-r^{2} \delta_{v}(P)-\frac{1}{2} v\left(\Psi_{r}^{2}(P)\right)+\frac{r^{2}-1}{12} v(\Delta)=0
$$

for any $P=\left(x_{P}, y_{P}\right) \in E(K)$ and $r \in \mathbf{N}$ such that $r P \neq \mathcal{O}$.

Proof. The proof is an adaptation of the corresponding proof of the existence theorem in [14]. Indeed, one exploits (10), (11) from Proposition 3 and (11') from the corollary to establish the existence of $\delta_{v, m}$. Then formulas (6) and (4) from the corollary to Proposition 1 are utilized to prove that $\delta_{v, m}$ fulfills the asserted relations 
(13) and (14). Finally, the independence of $\delta_{v, m}$ on $m$ is a consequence of the following

COROllary 1. The function $\delta_{v, m}$ on $E(K)$ is related to the local Weil height on $E(K)$ through the estimate

$$
\left|\delta_{v, m}(P)-\left\{d_{v}(P)+\frac{1}{12} v(\Delta)\right\}\right| \leqslant c_{m},
$$

where

$$
c_{m}=\frac{1}{m^{2}-1} \cdot \max \left\{\left|c_{1, m}\right|,\left|c_{2, m}\right|\right\}
$$

In fact, $\delta_{v, m}=\delta_{v}$ is uniquely determined by the properties (14) and (15) and hence is independent of the choice of $m$.

We can now define the global Neron-Tate height on $E(K)$ as the sum, with multiplicities, over the local Néron-Tate heights as follows (see [14]):

$$
\delta(P)= \begin{cases}\sum_{v \in M_{K}} \lambda_{v} \delta_{v}(P) & \text { if } P \neq \mathcal{O} \\ 0 & \text { if } P=\mathcal{O}\end{cases}
$$

By the sum formula (S) we then obtain on the basis of (13) and (14):

COROLlaRY 2. The global Néron-Tate height on $E(K)$ fulfills the relations

$$
\delta(P+Q)+\delta(P-Q)-2 \delta(P)-2 \delta(Q)=0
$$

and, for $r \in \mathbf{N}$,

$$
\delta(r P)-r^{2} \delta(P)=0
$$

Remark 2. Corollary 2 shows that the global Néron-Tate height $\delta$ is a quadratic form on $E(K)$, whereas Proposition 4 implies that the local Néron-Tate height $\delta_{v}$ is "almost" a quadratic form on $E(K)$.

4. Computation of the Néron-Tate Height. Again, let the elliptic curve $E$ be given by (E) over a global field $K$. Fix a nonarchimedean (discrete) valuation $v$ of $K$. Suppose that $P=\left(x_{P}, y_{P}\right) \in E(K)$ is a rational point satisfying $v\left(x_{P}\right)<\mu_{v}$.

By Proposition 1 , on choosing an $m \in \mathbf{N}$ such that $m \geqslant 2$ and $v(m)=0$, we have

$$
x_{m^{n} P}=\frac{\Phi_{m^{n}}(P)}{\Psi_{m^{n}}^{2}(P)} .
$$

Now $v\left(x_{P}\right)<\mu_{v}$ together with $v\left(a_{i}\right) \geqslant \mu_{v}$ entails

$$
v\left(\Phi_{m^{n}}(P)\right)=m^{2 n} v\left(x_{P}\right), \quad v\left(\Psi_{m^{n}}^{2}(P)\right)=\left(m^{2 n}-1\right) v\left(x_{P}\right) .
$$

Hence

$$
v\left(x_{m^{n} P}\right)=v\left(x_{P}\right)
$$


Thus we obtain from the limit formula (12) and the definition (9) of $d_{v}$ the asserted relation

$$
\delta_{v}(P)=-\frac{1}{2} v\left(x_{P}\right)+\frac{1}{12} v(\Delta)=d_{v}(P)+\frac{1}{12} v(\Delta)
$$

Proposition 5. Suppose that a rational point $P=\left(x_{P}, y_{P}\right) \in E(K)$ satisfies the inequality $v\left(x_{P}\right)<\mu_{v}$ for a nonarchimedean (discrete) valuation $v$ of the global field $K$. Then the local Neron-Tate height of $P$ essentially coincides with the local Weil height of $P$ with respect to $v$; more precisely,

$$
\delta_{v}(P)=d_{v}(P)+\frac{1}{12} v(\Delta) .
$$

From Proposition 5 we get the following theorem, which is crucial for the calculation of the Néron-Tate height on $E(K)$.

THEOREM 1. Let $E$ be an elliptic curve defined by a Weierstrass equation (E) over an algebraic number field $K$. Choose a discrete normalized additive valuation $v$ of $K$ and suppose that the equation (E) is minimal with respect to $v{ }^{*}$ Then, for each nontorsion point $P \in E_{0}(K)$, the local Neron-Tate height of $P$ is essentially equal to the local Weil height of $P$ with respect to $v$; more precisely,

$$
\delta_{v}(P)=d_{v}(P)+\frac{1}{12} v(\Delta) .
$$

Proof. The theorem can be found in [9]. For the convenience of the reader, however, we give a proof.

By Proposition 5, we may confine ourselves to the case in which $v\left(x_{P}\right) \geqslant \mu_{v}$. The subcase in which $v\left(x_{P}\right) \geqslant \mu_{v}>0$ would lead to a contradiction to the choice of $P \in E_{0}(K)$. Hence it remains to consider the subcase in which

$$
v\left(x_{P}\right) \geqslant \mu_{v}=0 \text {. }
$$

The reduction map of Section 2,

$$
\tilde{\rho}_{0}: E_{0}(K) / E_{1}(K) \rightarrow \tilde{E}_{0}(\tilde{K}),
$$

is an injective homomorphism. Since $K$ is a number field, the residue field $\tilde{K}$ of $K$ with respect to $v$ is finite and hence so is the group $\tilde{E}_{0}(\tilde{K})$. Therefore, for any $P \in E_{0}(K)$, there exists a number $r \in \mathbf{N}$ such that $r P \in E_{1}(K)$. Choose $r \in \mathbf{N}$ minimal with this property. Then we have

$$
v\left(x_{r P}\right)<\mu_{v}=0 .
$$

From this, since $v\left(x_{P}\right) \geqslant \mu_{v}=0$ and $v\left(a_{i}\right) \geqslant \mu_{v}=0$, we conclude that

$$
v\left(\Phi_{r}(P)\right) \geqslant 0 \text { and } v\left(\Psi_{r}(P)\right)>0 .
$$

We claim

$$
v\left(x_{r P}\right)=-v\left(\Psi_{r}^{2}(P)\right)
$$

\footnotetext{
* The required minimal model of $E$ is found by Tate's algorithm [11].
} 
By Proposition 5, Formula (14) of Proposition 4, and the definition (9) of $d_{v}$, this claim yields the asserted identity

$$
\begin{aligned}
\delta_{v}(P) & =\frac{1}{r^{2}}\left\{\delta_{v}(r P)-\frac{1}{2} v\left(\Psi_{r}^{2}(P)\right)+\frac{r^{2}-1}{12} v(\Delta)\right\} \\
& =d_{v}(P)+\frac{1}{12} v(\Delta)
\end{aligned}
$$

since $v\left(x_{P}\right) \geqslant \mu_{v}=0$.

To prove (17) it suffices to show that

$$
v\left(\Phi_{r}(P)\right)=0 .
$$

This is accomplished by verifying (18), first for the lower $r \in \mathbf{N}$ and then for general $r \in \mathbf{N}$.

Let $r=2$.

If $v\left(3 x_{P}^{2}+2 a_{2} x_{P}+a_{4}-a_{1} y_{P}\right)>0$ we would get a contradiction to the assumption that $P \in E_{0}(K)$. Hence it is enough to consider $v\left(3 x_{P}^{2}+2 a_{2} x_{P}+a_{4}-a_{1} y_{P}\right)$ $=0$. But then the asserted relation (18) follows directly from the formula (2) for $x_{2 P}$ and Proposition 1.

Let $r=3$.

By the minimal choice of $r$, we have

$$
v\left(\Psi_{2}(P)\right)=0 \text { and } \quad v\left(\Psi_{3}(P)\right)>0 .
$$

Now the decomposition formula (which can be verified without trouble)

$$
\Psi_{4}(P)=\Psi_{2}(P)\left[\Psi_{3}(P)\left(6 x_{P}^{2}+b_{2} x_{P}+b_{4}\right)-\Psi_{2}^{4}(P)\right]
$$

yields $v\left(\Psi_{4}(P)\right)=0$, and hence the relation from Proposition 1,

$$
\Phi_{3}(P)=x_{P} \Psi_{3}^{2}(P)-\Psi_{2}(P) \Psi_{4}(P),
$$

leads to the identity $v\left(\Phi_{3}(P)\right)=0$, as asserted in (18).

Finally, let $r \geqslant 4$.

Again, by the choice of $r$, we have

$$
\begin{aligned}
& v\left(\Psi_{2}(P)\right)=v\left(\Psi_{3}(P)\right)=\cdots=v\left(\Psi_{r-1}(P)\right)=0 \quad \text { and } \\
& v\left(\Psi_{r}(P)\right)>0 .
\end{aligned}
$$

Then Formula (5) from the corollary to Proposition 1 yields

$$
v\left(x_{2 P}-x_{P}\right)=0 \text { and } v\left(x_{(r-1) P}-x_{P}\right)>0,
$$

so that another consequence of Formula (5), viz.,

$$
\Psi_{r+1}(P)=-\left[\left(x_{(r-1) P}-x_{P}\right)+\left(x_{P}-x_{2 P}\right)\right] \frac{\Psi_{r-1}^{2}(P) \Psi_{2}^{2}(P)}{\Psi_{r-3}(P)},
$$

leads to $v\left(\Psi_{r+1}(P)\right)=0$. Now the identity from Proposition 1 ,

$$
\Phi_{r}(P)=x_{P} \Psi_{r}^{2}(P)-\Psi_{r-1}(P) \Psi_{r+1}(P),
$$

reveals that $v\left(\Phi_{r}(P)\right)=0$, as asserted in (18). This proves Theorem 1 . 
Remark 3. Theorem 1 makes it possible to calculate the local Néron-Tate height $\delta_{v}(P)$ with respect to all discrete valuations $v$ of the number field $K$ for all nontorsion points $P \in E(K)$.

This is true because Proposition 2 tells us that a suitable multiple $r P$ of $P$ belongs to $E_{0}(K)$. Then we apply Theorem 1 to calculate $\delta_{v}(r P)$ and use Formula (14) from Proposition 4 to get the desired value of $\delta_{v}(P)$ itself.**

Remark 4. Torsion points $P \in E(K)$ are of no interest in this connection since their global Néron-Tate height is $\delta(P)=0$.

It remains to show how to compute the local Néron-Tate height $\delta_{v}$ for archimedean valuations $v$ of the number field $K$. From $\left(4^{\prime}\right)$ in the corollary to Proposition 1 , we get the formula

$$
\frac{1}{2} \frac{v\left(\Psi_{m^{n}}^{2}(P)\right)}{m^{2 n}}=\sum_{\nu=1}^{m} \frac{1}{2} \frac{v\left(\Psi_{m}^{2}\left(m^{\nu-1} P\right)\right)}{m^{2 \nu}}
$$

which proves to be useful in the sequel.

Now, since we are interested here only in the case of $K=\mathbf{Q}$, the field of rational numbers, we confine ourselves to considering its completion $K_{\infty}=\mathbf{Q}_{\infty}=\mathbf{R}$ with respect to the ordinary absolute value $v=v_{\infty}=-\log ||$. Then Tate's method is best suited for calculating $\delta_{v_{\infty}}$ (see [12]).

THEOREM 2. Let $E$ be an elliptic curve defined by a Weierstrass equation (E) over the field $\mathbf{R}$ of real numbers and denote by $v_{\infty}=-\log ||$ the ordinary additive archimedean valuation of $\mathbf{R}$. Take an open subgroup $\Gamma$ of $E(\mathbf{R})$ such that all $P=\left(x_{P}, y_{P}\right) \in \Gamma$ satisfy $x_{P} \neq 0 .{ }^{* * *}$ For $P \in \Gamma$ such that $2^{n} P \neq \mathcal{O}$ for all $n \in \mathbf{N}$, define the entities $T_{n}, W_{n}$, and $Z_{n}$ by putting

$$
T_{0}=\frac{1}{x_{P}}, \quad T_{n+1}=\frac{W_{n}}{Z_{n}} \quad \text { for } n \in \mathbf{N}_{0},
$$

where

$$
W_{n}=4 T_{n}+b_{2} T_{n}^{2}+2 b_{4} T_{n}^{3}+b_{6} T_{n}^{4}, \quad Z_{n}=1-b_{4} T_{n}^{2}-2 b_{6} T_{n}^{3}-b_{8} T_{n}^{4} .
$$

Let

$$
\mu(P)=\sum_{n=0}^{\infty} \frac{\log \left|Z_{n}\right|}{2^{2 n}}, \quad \lambda(P)=\frac{1}{2} \log \left|x_{P}\right|+\frac{1}{8} \mu(P) .
$$

Then the local Neron-Tate height of $P$ with respect to $v_{\infty}$ is

$$
\delta_{v_{\infty}}(P)=\lambda(P)-\frac{1}{12} \log |\Delta| \text {. }
$$

Proof. See [12]. However, the assertion of Theorem 2 also follows from

Proposition 6. In the situation of Theorem 2 we have for $n \in \mathbf{N}_{0}$,

$$
T_{n}=\frac{1}{x_{2^{n} P}}, \quad W_{n}=\frac{\Psi_{2}^{2}\left(2^{n} P\right)}{x_{2^{n} P}^{4}}, \quad Z_{n}=\frac{\Phi_{2}\left(2^{n} P\right)}{x_{2^{n} P}^{4}} .
$$

** Added in proof. Joe Silverman, whom we wish to thank for some valuable hints, told us that he has carried out similar height computations (unpublished) avoiding, however, the use of Proposition 2 by employing Tate's local formulas (see [14]).

${ }^{* * *}$ Hence $\Gamma$ is either $E(\mathbf{R})$ or the identity component of $E(\mathbf{R})$ according as $E(\mathbf{R})$ is connected or disconnected. 
Proof. The proof is carried out easily by means of the formulas (7) in the corollary to Proposition 1.

Remark 5. The simplest way of finding a subgroup $\Gamma$ of $E(\mathbf{R})$ of the type desired in Theorem 2 is by applying a birational transformation to (E) to obtain a model $\left(\mathrm{E}^{\prime}\right)$ such that $b_{6}^{\prime}<0$. Then $\Gamma=E^{\prime}(\mathbf{R})$ itself will do.

In the special case of $K=\mathbf{Q}$ we are interested in, the set $M_{\mathbf{Q}}$ consists in the $p$-adic valuations $v_{p}$ corresponding to the primes $p$ of $\mathbf{Q}$ and the additive valuation $v_{\infty}=-\log ||$ corresponding to the unique archimedean absolute value || on $\mathbf{Q}$. Of course, the multiplicities in the sum formula $(\mathrm{S})$ are all $\lambda_{v}=1$.

5. Examples. We are now in a position to calculate the Néron-Tate height $\delta$ on the group $E(K)$ of rational points on an elliptic curve $E$ over the rational number field $K=\mathbf{Q}$. To this end, we use the defining formula (16) for $\delta$ with multiplicities $\lambda_{v}=1$ to reduce the computation of $\delta$ to that of the local Néron-Tate heights $\delta_{v}$ on $E(K)$. For discrete valuations $v$ of $\mathbf{Q}$, the height $\delta_{v}$ is calculated by means of Theorem 1 in accordance with Remark 3, and for the archimedean absolute value $v_{\infty}=-\log \mid$ |, the calculation of $\delta_{v_{\infty}}$ is performed on the basis of Theorem 2 .

(i) Examples of Silverman. We illustrate our procedure by verifying the height calculations of Silverman [9].

$$
\begin{aligned}
& E: y^{2}+21 x y+494 y=x^{3}+26 x^{2}, \\
& P=(0,0) \in E(\mathbf{Q}), \\
& \Delta=-2^{13} \cdot 13^{3} \cdot 19^{2} .
\end{aligned}
$$

Silverman obtains

$$
\delta(P)=0.010,492, \ldots
$$

We have

$$
\delta_{v_{\infty}}(P)=0.038,612,393, \ldots,
$$

$$
P \notin E_{0}(\mathbf{Q}) \text { for } p=2,13 \text { and } 19 \text {; and }
$$

$$
\delta_{v_{p}}(P)=0 \text { for all primes } p \neq 2,13 \text { or } 19 \text {. }
$$

Now

$$
\begin{array}{ll}
13 P \in E_{0}(\mathbf{Q}) & \text { for } p=2, \\
3 P \in E_{0}(\mathbf{Q}) & \text { for } p=13, \\
2 P \in E_{0}(\mathbf{Q}) & \text { for } p=19
\end{array}
$$

One computes

$$
\Psi_{2}(P)=2 \cdot 13 \cdot 19, \quad \Psi_{3}(P)=2^{3} \cdot 13^{3} \cdot 19^{2}, \quad \Psi_{13}(P)=-2^{80} \cdot 13^{56} \cdot 19^{42}
$$

and

$$
x_{2 P}=-2 \cdot 13, \quad x_{3 P}=-2 \cdot 19, \quad x_{13 P}=-2^{4} \cdot 5 \cdot 13 \cdot 19 .
$$

This leads to

$$
\delta_{v_{2}}(13 P)=\frac{37}{12} \ln 2, \quad \delta_{v_{13}}(3 P)=\frac{1}{4} \ln 13, \quad \delta_{v_{19}}(2 P)=\frac{1}{6} \ln 19 .
$$

Hence, by (14) of Proposition 4,

$$
\delta_{v_{2}}(P)=\frac{97}{156} \ln 2, \quad \delta_{v_{13}}(P)=-\frac{1}{12} \ln 13, \quad \delta_{v_{19}}(P)=-\frac{1}{12} \ln 19 .
$$


By (16) this adds up to

$$
\begin{gathered}
\delta(P)=0.010,492,061, \ldots \\
E: y^{2}+11 x y+80 y=x^{3}+8 x^{2}, \\
P=(0,0) \in E(\mathbf{Q}), \\
\Delta=-2^{11} \cdot 5^{2} \cdot 19 .
\end{gathered}
$$

Silverman gets

$$
\delta(P)=0.010,284, \ldots
$$

and we obtain similarly to (A)

$$
\delta(P)=0.010,284,005, \ldots
$$

(ii) The Bremner-Cassels Curves. Our procedure turns out to be particularly useful for calculating the global Néron-Tate height on the elliptic curves

$$
E_{p}: y^{2}=x^{3}+p x
$$

for primes $p$ of $\mathbf{Q}$ such that $p \equiv 5(\bmod 8)$, as they were considered by Bremner and Cassels [1]. The authors exhibit points $P \in E(\mathbf{Q})$ of infinite order on 43 curves of this type, where

$$
P=\left(x_{P}, y_{P}\right) \quad \text { with } x_{P}=\frac{r^{2}}{s^{2}}, \quad y_{P}=\frac{r \cdot t}{s^{3}} \quad \text { for } r, s, t \in \mathbf{Z}
$$

such that

$$
\begin{aligned}
& \text { g.c.d. }(r, s)=1 ; \quad r, t \neq 0(\bmod p) ; \quad \text { and } \\
& r \equiv t \equiv 1(\bmod 2), \quad s \equiv 0(\bmod 2) .
\end{aligned}
$$

One easily checks that $P \in E_{0}(\mathbf{Q})$ for all primes $p$ of $\mathbf{Q}$ and all points $P \in E(\mathbf{Q})$ displayed in [1]. (Notice that $2 y_{P}$ and $3 x_{P}^{2}+p$ are relatively prime.) This leads to

Proposition 7. For the points $P \in E_{p}(\mathbf{Q})$ of infinite order on the Bremner-Cassels curves in [1], the Neron-Tate height is

$$
\delta(P)=\delta_{v_{\infty}}(P)+\frac{1}{12} \ln |\Delta|+\ln |s|
$$

(iii) Modular Elliptic Curves. In [16, pp. 75-113], N. M. Stephens and J. Davenport list 68 modular elliptic curves $E$ of rank 1 with a rational point $P \in E(\mathbf{Q})$ of infinite order. We computed the Néron-Tate heights of these points $P .^{\dagger}$ Comparison of the Neron-Tate height of the generator of the 63rd curve in their table with the Néron-Tate height of the point in Silverman's second example (see (i) (B) above) shows that the two values agree. It turns out, as one easily checks, that the corresponding two curves are birationally isomorphic (see Table 1).

\footnotetext{
† We have compared the height values in our Table 1 with those in a corresponding (unpublished) table of Silverman containing up to six digits behind the period. They agree (except for the sixth digits of the curves 58A, 61A, 135A, 153A, 189C and for the fifth and sixth digit of the curve 185D).
} 
TABLE 1

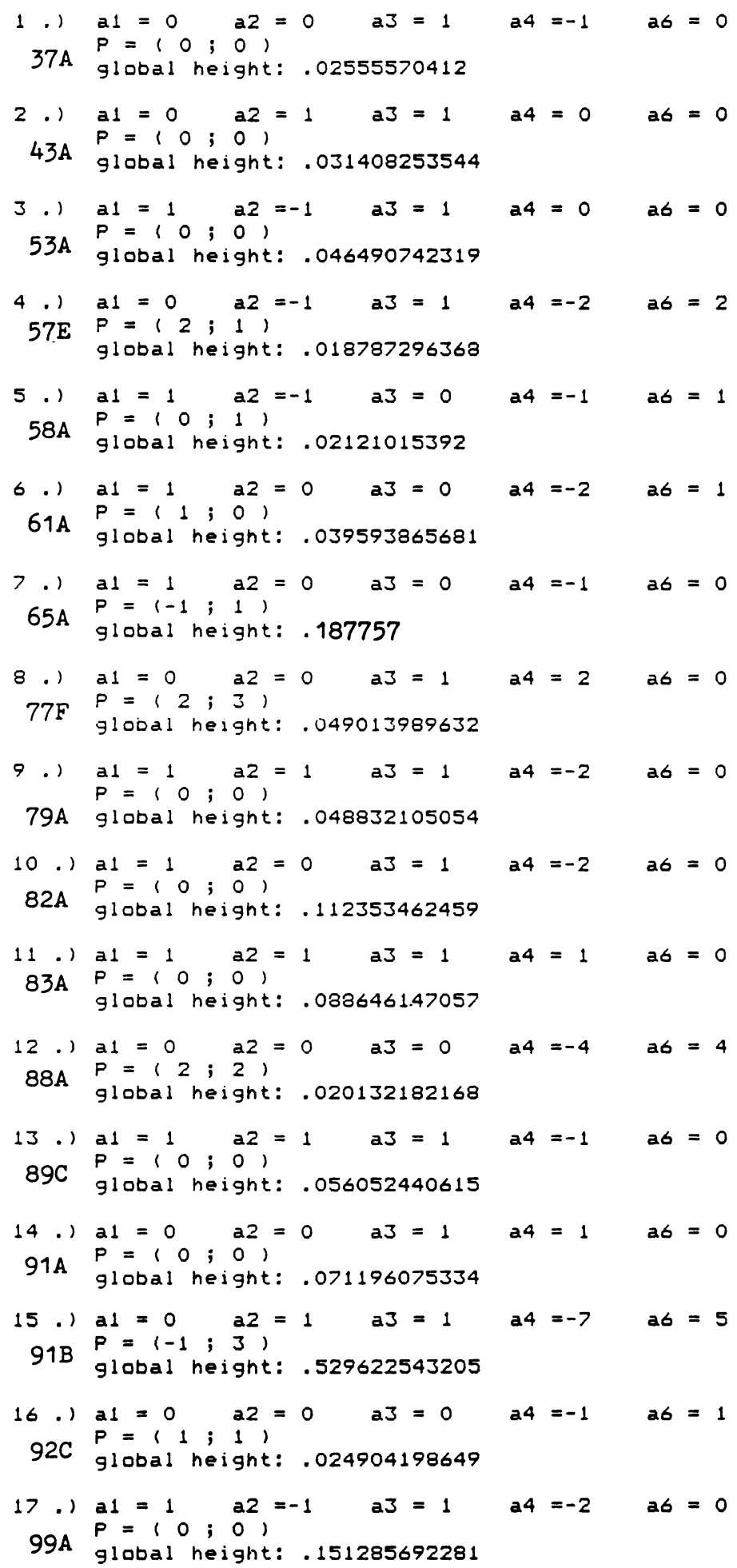


TABLE 1 (continued)

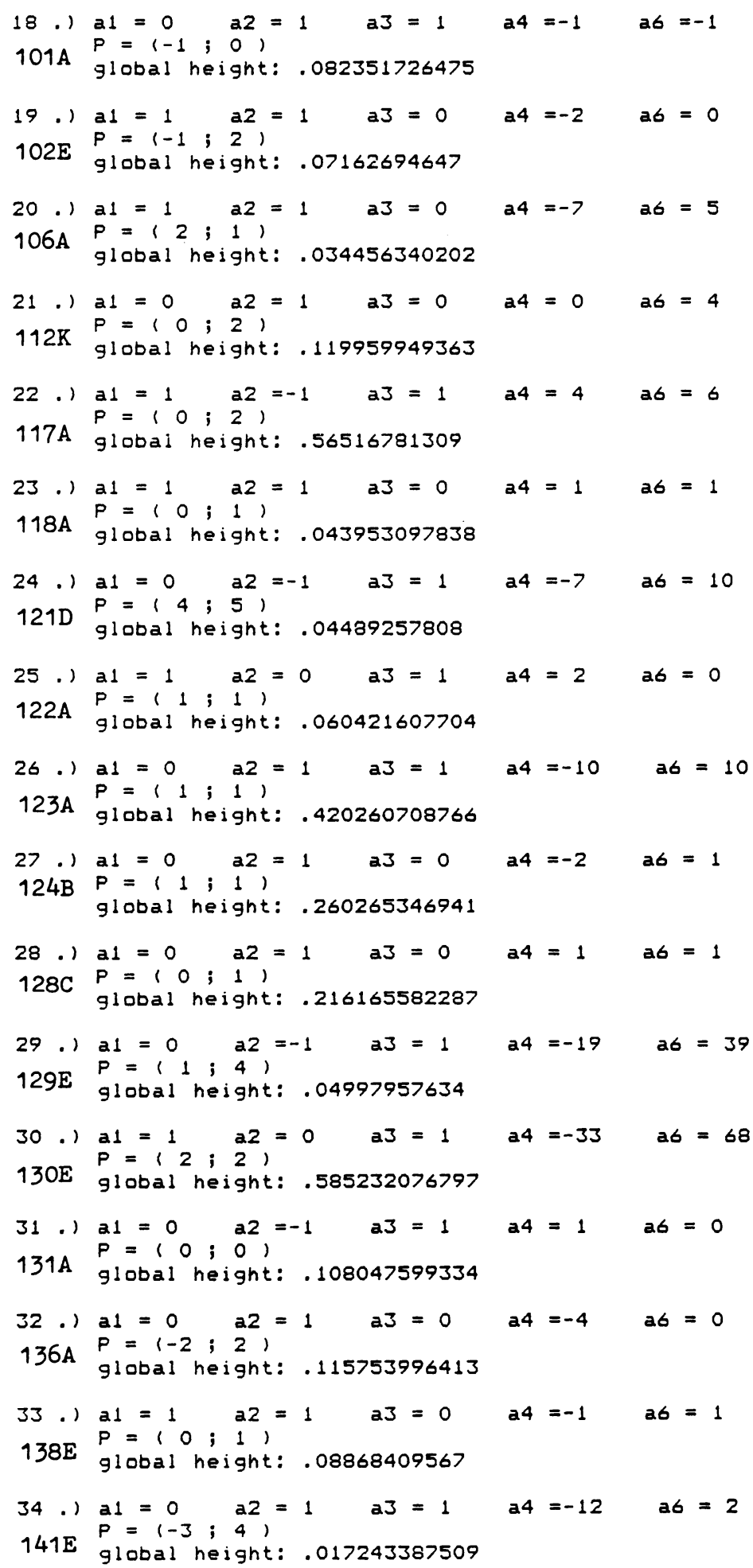




\section{TABLE 1 (continued)}

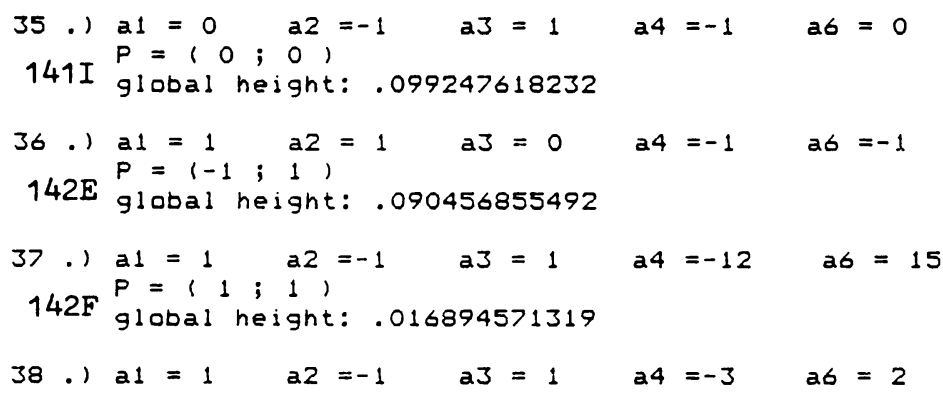


TABLE 1 (continued)

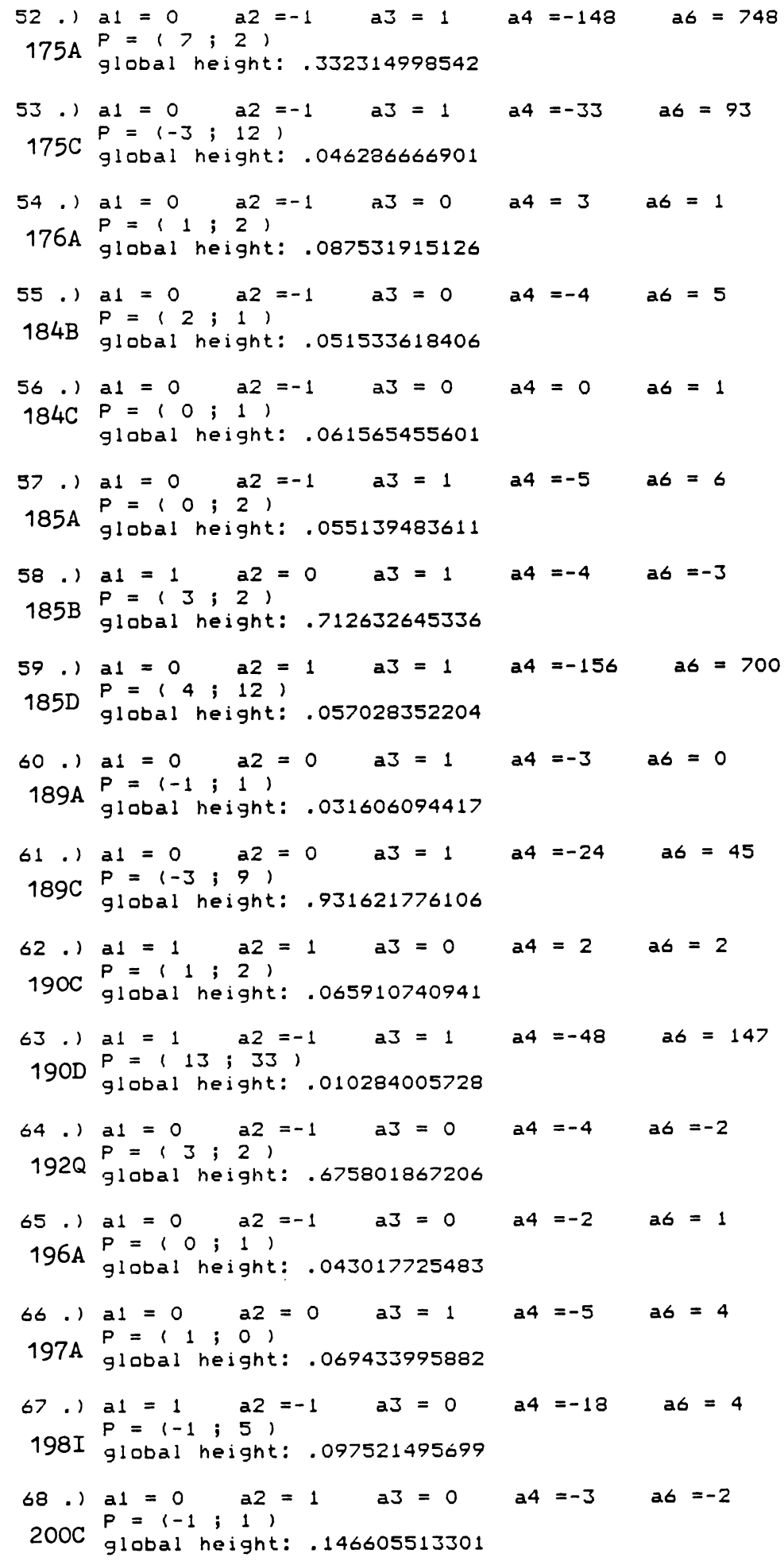


6. Lang's Conjectures. Silverman [9] used his above-cited examples of rank-one elliptic curves $E$ over $\mathbf{Q}$ to estimate the constants $c_{1}, c_{2}$ in S. Lang's Conjecture 2 (see [6]) about lower bounds for the Néron-Tate height $\delta$ on nontorsion points in $E(\mathbf{Q})$. We wish to carry through a similar estimation with respect to Lang's Conjecture 3 (see [6]) for Selmer's [8] rank-two elliptic curves $E$ over $\mathbf{Q}$.

In Section 3, Remark 2, we observed that the Néron-Tate height $\delta$ is a quadratic form on the rational point group $E(\mathbf{Q})$. This property of $\delta$ is tantamount to the fact that the function

$$
\beta(P, Q)=\frac{1}{2}\{\delta(P+Q)-\delta(P)-\delta(Q)\}
$$

\section{TABLE 2}

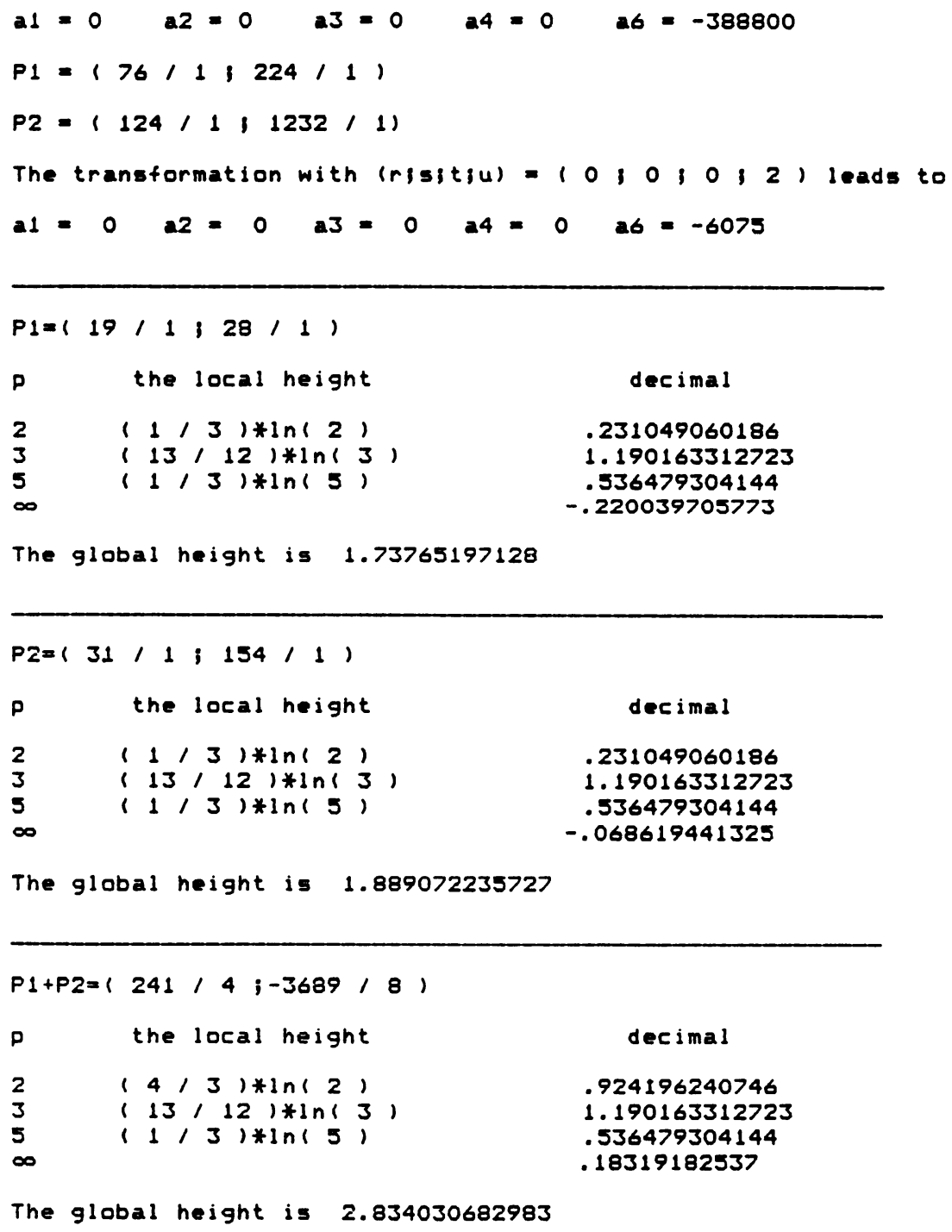

The global height is 2.834030682983 
In analogy to Silverman [9], we now use these Selmer curves to estimate the constants in Lang's Conjecture 3. Suppose $E$ over $\mathbf{Q}$ is given in Weierstrass normal form

$$
y^{2}=x^{3}+a x+b \quad(a, b \in \mathbf{Z}) .
$$

Following Lang [6], we define the height of $E$ over $\mathbf{Q}$ to be the number

$$
H(E)=\max \left\{|a|^{3},|b|^{2}\right\},
$$

so that approximately

$$
h(E)=-\log H(E) \approx 6 \mu_{v_{\infty}},
$$

where again $v_{\infty}=-\log ||$ denotes the additive archimedean valuation of $\mathbf{Q}$. Let $N$ stand for the conductor of $E$ over $\mathbf{Q}$ (see [11]).

Then we enunciate, in the case of rank-two curves,

LANG's Conjecture 3. There is a basis $\left\{P_{1}, P_{2}\right\}$ of $E(\mathbf{Q})$ modulo torsion such that $\delta\left(P_{1}\right) \leqslant \delta\left(P_{2}\right)$ and

$$
\begin{aligned}
& \delta\left(P_{1}\right) \leqslant c_{1} H(E)^{1 / 24} \cdot N^{\varepsilon(N) / 2} \cdot \log N \cdot(2 / \sqrt{3})^{1 / 2}, \\
& \delta\left(P_{2}\right) \leqslant c_{2} H(E)^{1 / 12} \cdot N^{\varepsilon(N)} \cdot \log N \cdot c
\end{aligned}
$$

for some positive real constants $c, c_{1}, c_{2}$, where

$$
\lim _{N \rightarrow \infty} \varepsilon(N)=0 \text {. }
$$

Now the constants $c_{1}$ and $c_{2}$ in Lang's Conjecture 3 satisfy the inequalities

$$
\begin{aligned}
& c_{1} \geqslant\left(\frac{H(E)^{1 / 24} \cdot N^{\varepsilon(N) / 2} \cdot \log N \cdot(2 / \sqrt{3})^{1 / 2}}{\delta\left(P_{1}\right)}\right)^{-1}, \\
& c_{2} \geqslant\left(\frac{H(E)^{1 / 12} \cdot N^{\varepsilon(N)} \cdot \log N \cdot c}{\delta\left(P_{2}\right)}\right)^{-1} .
\end{aligned}
$$

On choosing $c=1$ and putting, in analogy to the example on p. 166 of [6],

$$
\varepsilon(N)=(\log N \cdot \log \log N)^{-1 / 2},
$$

we obtain for the constants $c_{1}$ and $c_{2}$ the estimates ${ }^{\dagger \dagger}$

$$
c_{1} \geqslant 0.021,784, \ldots, \quad c_{2} \geqslant 0.002,709, \ldots
$$

Here we let $E$ range over the rank-two curves in [8] and take the maximal values for $c_{1}$ and $c_{2}$, which are attained at the curves with $A=246$ and $A=30$, respectively. For the sake of completeness, we include here the numerical estimates of the constants $c_{1}$ and $c_{2}$ for all values of $A$ in Selmer's table [8] in order to show how $c_{1}$ and $c_{2}$ oscillate as $A$ varies (see Table 3 ).

\footnotetext{
it This estimation is based on the assumption that the points in Selmer's table [8] are of minimal height. We wish to thank M. Reichert for verifying this on a Siemens PC MX-2 for Selmer's curves with $A=30$, $37,65,91,110,124,126,163,182,217,254,342,468$ and 469 . Only for $A=254$, the point $P_{1}+P_{2}$ is to be taken instead of $P_{2}$ since it has a slightly smaller height value.
} 
TABLE 3

\begin{tabular}{|c|c|c|}
\hline 19 & 0.00513126 & 0.00110389 \\
\hline 30 & 0.0192703 & 0.00270903 \\
\hline 37 & 0.00483937 & 0.000962382 \\
\hline 65 & 0.00345923 & 0.00182501 \\
\hline 86 & 0.00629273 & 0.00227975 \\
\hline 91 & 0.0036522 & 0.000438337 \\
\hline 110 & 0.012277 & 0.00153855 \\
\hline 124 & 0.00374265 & 0.00174993 \\
\hline 126 & 0.00433063 & 0.00114267 \\
\hline 127 & 0.00414683 & 0.000940948 \\
\hline 132 & 0.0183099 & 0.00244798 \\
\hline 153 & 0.0105335 & 0.00111188 \\
\hline 163 & 0.00458141 & 0.000483074 \\
\hline 182 & 0.00445021 & 0.000517017 \\
\hline 183 & 0.00524747 & 0.00199503 \\
\hline 201 & 0.00511671 & 0.00120915 \\
\hline 203 & 0.0095474 & 0.00116455 \\
\hline 209 & 0.00721788 & 0.000812224 \\
\hline 210 & 0.0126048 & 0.00121204 \\
\hline 217 & 0.00308153 & 0.000319989 \\
\hline 218 & 0.00327199 & 0.00201074 \\
\hline 219 & 0.00500126 & 0.00171349 \\
\hline 246 & 0.0217843 & 0.00193905 \\
\hline 254 & 0.00531365 & 0.000936257 \\
\hline 271 & 0.00370666 & 0.000782067 \\
\hline 273 & 0.00472123 & 0.000663038 \\
\hline 282 & 0.0182864 & 0.00183782 \\
\hline 309 & 0.00457362 & 0.00227242 \\
\hline 335 & 0.00274443 & 0.00165495 \\
\hline 342 & 0.00352578 & 0.00103604 \\
\hline 345 & 0.0142214 & 0.00141568 \\
\hline 348 & 0.0175728 & 0.0019122 \\
\hline 370 & 0.00282848 & 0.00117946 \\
\hline 379 & 0.0038473 & 0.00071123 \\
\hline 390 & 0.0111818 & 0.00085906 \\
\hline 397 & 0.00349161 & 0.000596949 \\
\hline 399 & 0.0042891 & 0.000624869 \\
\hline 407 & 0.00267502 & 0.00118686 \\
\hline 420 & 0.0121164 & 0.00107526 \\
\hline 433 & 0.00518512 & 0.000522935 \\
\hline 435 & 0.0139496 & 0.00147541 \\
\hline 436 & 0.00482868 & 0.00160066 \\
\hline 446 & 0.0041804 & 0.00145041 \\
\hline 453 & 0.00415706 & 0.00168546 \\
\hline 462 & 0.0108269 & 0.000877993 \\
\hline 468 & 0.00388973 & 0.00098101 \\
\hline 469 & 0.00339982 & 0.00030445 \\
\hline 477 & 0.00744997 & 0.00111861 \\
\hline 497 & 0.00531293 & 0.00090782 \\
\hline & 0.0171181 & 0.0014825 \\
\hline
\end{tabular}

Fachbereich 9 Mathematik

Universität des Saarlandes

D-6600 Saarbrücken, West Germany

1. A. Bremner \& J. W. S. Cassels, "On the equation $Y^{2}=X^{2}(X+p)$," Math. Comp., v. 42, 1984. pp. 257-264.

2. J. P. Buhler, B. H. Gross \& D. B. Zagier, "On the conjecture of Birch and Swinnerton-Dyer for an ellliptic curve of rank 3," Math. Comp., v. 44, 1985, pp. 473-481.

3. J. W. S. Cassels, "Diophantine equations with special reference to elliptic curves," J. London Math. Soc., v. 41, 1966, pp. 193-291.

4. H. G. Folz, Ein Beschränktheitssatz für die Torsion von 2-defizienten elliptischen Kurven über algebraischen Zahlkörpern, Ph.D. Thesis, Saarbrücken, 1985. 
5. S. LaNG, Elliptic Curves: Diophantine Analysis, Springer-Verlag, Berlin and New York, 1978.

6. S. LANG, “Conjectured Diophantine estimates on elliptic curves," Progr. Math., v. 35, 1983, pp. $155-171$.

7. S. LANG, Fundamentals of Diophantine Geometry, Springer-Verlag, Berlin and New York, 1983.

8. E. Selmer, “The Diophantine equation $a x^{3}+b y^{3}+c z^{3}$," Acta Math., v. 85, 1951, pp. 203-362.

9. J. H. Silverman, "Lower bound for the canonical height on elliptic curves," Duke Math. J., v. 48, 1981, pp. 633-648.

10. J. T. TATE, “The arithmetic of elliptic curves," Invent. Math., v. 23, 1974, pp. 179-206.

11. J. T. TATE, "Algorithm for finding the type of a singular fibre in an elliptic pencil," in Modular Functions of One Variable IV, Lecture Notes in Math., vol. 476, Springer-Verlag, Berlin and New York, 1975, pp. 33-52.

12. J. T. TATE, Letter to J.-P. Serre, Oct. 1, 1979.

13. H. G. ZIMMER, "On the difference of the Weil height and the Néron-Tate height," Math. Z., v. 147 , 1976, pp. 35-51.

14. H. G. Zimmer, "Quasifunctions on elliptic curves over local fields," J. Reine Angew. Math., v. $307 / 308,1979$, pp. 221-246.

15. H. G. ZIMMER, “Torsion points on elliptic curves over a global field," Manuscripta Math., v. 29 , 1979, pp. 119-145.

16. Modular Functions of One Variable IV (B. J. Birch \& W. Kuyk, eds.), Lecture Notes in Math., vol. 476, Springer-Verlag, Berlin and New York, 1975. 